REVESCO. Revista de Estudios Cooperativos

ISSN: $1885-8031$

http://dx.doi.org/10.5209/REVE.58396

\title{
Modificaciones estructurales en cooperativas. La transformación de una cooperativa en sociedad limitada y la determinación del momento del cálculo y del importe de los fondos a reintegrar. Un caso práctico
}

\author{
Miguel Ángel Crespín García ${ }^{1}$ y Juan Vicente Fruet Cardozo ${ }^{2}$
}

Recibido: 14 de julio de 2017 / Aceptado: 27 de octubre de 2017

Resumen. El presente artículo tiene como objetivo dar claridad a la problemática que surge al realizar la transformación de una sociedad cooperativa en sociedad limitada, en cuanto al momento del cálculo e importe de fondos a reintegrar. Se presenta un caso práctico del proceso de transformación de una sociedad cooperativa en una sociedad limitada, donde se analiza, además del proceso mencionado, la problemática práctica que surge en su desarrollo. Al final del mismo se incluyen las conclusiones sobre el procedimiento.

Palabras clave: Sociedad cooperativa andaluza; Transformación; Fondos sociales; Fiscalidad y Contabilidad.

Claves Econlit: M00; M40; K00.

[en] Structural modifications in cooperatives. The transformation of a cooperative in a limited partnership and determination of the calculation and amount of funds to reimburse. A practical case

\begin{abstract}
The purpose of this article is to clarify the problems arising from the transformation of a cooperative society into a limited partnership, as to the timing of the calculation and amount of funds to be reinvested. In the present paper,it is presented a practical case: the process of transformation of a cooperative society into a limited society, where, in addition to the aforementioned process, the practical problem that arises in its development is analyzed. The conclusions of the procedure are included at the end.
\end{abstract}

Keywords: Andalusian cooperative society; Transformation; Social funds; Taxation and Accounting.

Sumario. 1. Introducción. 2. Proceso de transformación de una cooperativa en una sociedad limitada, según la ley andaluza de cooperativas. 3. Conclusiones. 4. Referencias bibliográficas.

Cómo citar: Crespín García, M.A. y Fruet Cardozo, J.V. (2018) Modificaciones estructurales en cooperativas. La transformación de una cooperativa en sociedad limitada y la determinación del momento del cálculo y del importe de los fondos a reintegrar. Un caso práctico. REVESCO. Revista de Estudios Cooperativos, Primer Cuatrimestre, N 127, pp. 70-89. DOI: 10.5209/REVE.58396.

1 Universidad de Córdoba, España

Dirección de correo electrónico: es1crgam@uco.es.

2 Universidad de Córdoba, España

Dirección de correo electrónico: es1frcaj@uco.es. 


\section{Introducción}

\subsection{Objetivo del trabajo}

El objetivo de este trabajo se centra en una materia escasamente estudiada hasta la fecha en el ámbito económico-financiero, fiscal y contable, como es la determinación del momento e importe de los fondos sociales a reintegrar en las transformaciones de sociedades cooperativas en otro tipo de formas jurídicas; en concreto, en sociedad limitada.

Después de realizar un análisis detallado de todo lo publicado en materia de transformación de cooperativas, comprobamos que existen artículos que analizan aspectos de la transformación desde un punto de vista jurídico, fiscal y contable, que serán citados más adelante. Pero el tema de cómo y cuando calcular los fondos a reintegrar en los procesos de transformación, se estudia muy limitadamente, tratándose de un aspecto que en la práctica diaria está siendo cada vez más habitual y causa verdaderos inconvenientes, para todos los estamentos involucrados. De esta manera, nos encontramos con actuaciones de la Administración que difieren de unas sociedades a otras, percibiendo la falta de un procedimiento estándar a aplicar, lo que conlleva un auténtico problema a las entidades que deciden llevar a cabo dicho proceso y que, incluso, puede desembocar en la negativa por parte de la Administración a la concesión de la baja en el registro de cooperativas, por discrepancias surgidas en el ingreso de los fondos realizados.

\subsection{Revisión bibliográfica}

En relación a los estudios realizados sobre la materia analizada se puede citar el artículo Rojo Ramírez, Esteban Cerdán, Sánchez Pérez (2001: 57-76) que ya contemplaba la problemática de la determinación del importe de los fondos, según la Ley 27/1999 de Cooperativas y por las distintas leyes autonómicas, como es el caso de la andaluza. Al final del mismo, se logra interpretar la confusa redacción establecida en la legislación vigente en aquel momento, aplicándose a un caso real de transformación de sociedad cooperativa y la determinación de los fondos a reintegrar a la Junta de Andalucía a consecuencia de la citada transformación.

También cabe destacar el trabajo realizado por Nagore (2001) donde se trata la problemática de la transformación bajo el prisma de la legislación estatal, aportando valiosas soluciones al proceso.

Existen otros trabajos sobre la transformación de cooperativas, donde se analiza con detalle el aspecto legal de los procesos de reestructuración en cooperativas, tales como: (i) Vidal Portabales (1997: 217-248) que analiza la transformación de la cooperativa en otro tipo de sociedades, entre ellas la limitada, no obstante, se centra más en la posición jurídica de los socios y los acreedores que en la problemática de los fondos en dicho proceso. (ii) Nagore (2010: 47-50) que trata la problemática del mantenimiento de derecho político y económico del socio cooperativista después de la transformación. (iii) Alonso (2000: 75-113) hace un análisis jurídico de la legislación de reestructuración en cooperativas. (iv) León Sanz (1997: 25-60) analiza el marco jurídico de la transformación y otras modificaciones estructurales en cooperativas; (v) Rodríguez Artigas (2001: 13-38); 
(vi) Rodríguez Artigas (2009); (vi) Macías Ruano (2002: 51-88); (vii) Alfonso Sánchez (1997: 178-193).

Por último, también existen otros trabajos donde se analizan los aspectos contables y fiscales del proceso de transformación. Los principales son: (i) Bahía Almansa (2007); (ii) Bahía Almansa (2010: 93 - 119); y, (iii) Polo Garrido (2006: 108-138).

\subsection{Metodología utilizada}

El artículo utiliza la metodología del enfoque cuantitativo en las ciencias sociales, basada en el denominado "positivismo" . Este criterio sostiene que las ciencias sociales pueden estudiarse de manera similar al mundo natural; es decir, tal como se investigan los átomos, los planetas, los invertebrados, etc., también se pueden analizar los patrones de conducta de los trabajadores en una empresa, las migraciones humanas, e incluso, lo que nos ocupa en este trabajo: la transformación de una sociedad cooperativa en una sociedad limitada. Para el positivismo, la objetividad es clave; el investigador observa, mide y manipula variables y lo que no puede medirse u observarse con precisión, se descarta como objeto de estudio. Sólo se aceptan conocimientos que proceden de la experiencia, esto es, de datos empíricos; en nuestro caso, básicamente, los estados económicofinancieros de una sociedad cooperativa y la legislación andaluza.

Dentro de este contexto, el caso que nos ocupa es un hecho económico real, donde se presentan unos estados financieros y se desarrolla la transformación de una cooperativa en una sociedad limitada, según la legislación andaluza. Para ello se han considerado los aspectos fiscales, contables y mercantiles de la operación. En dicho caso práctico se determina el importe de los fondos a reintegrar y el momento de su cálculo. Por último, se cierra el trabajo con unas conclusiones sobre cómo se podría mejorar el proceso analizado.

\section{Proceso de transformación de una cooperativa en una sociedad limitada, según la ley andaluza de cooperativas}

"La transformación de sociedades cooperativas, es una operación social compleja, tanto por la diversidad de aspectos implicados (mercantil, fiscal y contable), así como por la importancia de los intereses en juego para socios, acreedores y terceros en general. Pero la transformación de sociedades cooperativas plantea, además, algunas dificultades adicionales. Éstas derivan, por un lado, de cuestiones jurídico-societarias, y por otro, de la complejidad y diversidad de las legislaciones implicadas en su regulación" ${ }^{4}$.

3 El positivismo nace con Augusto Comte (1798-1857) y Emile Durkheim (1858-1917), con la influencia significativa de Francis Bacon, John Locke y Emmanuel Kant, este último considerado el principal artífice de esta escuela científica. Con posterioridad, fue reemplazado por el "pospositivismo".

4 Bahía Almansa, B. (2010), Una aproximación a la fiscalidad de la transformación en el seno de las sociedades cooperativas. GEZKI, Nº $6,96$. 
La sociedad que vamos a modificar estructuralmente en este caso práctico, es una cooperativa andaluza de trabajo asociado, la cual, se va a transformar en una sociedad limitada. Según la Ley 3/2009, de 3 de abril, sobre Modificaciones Estructurales de las sociedades mercantiles se entiende que "una sociedad cooperativa podrá transformarse en sociedad mercantil, y una sociedad mercantil inscrita en sociedad cooperativa". Por lo tanto, es esencial determinar qué ocurre cuando se decide llevar a cabo el proceso de transformación de cooperativa a sociedad limitada. En el caso que nos ocupa, la legislación que resultaría aplicable será la Ley 14/2011 y de manera supletoria la Ley 27/1999. Por otro lado, todo el proceso de transformación está desarrollado en el Real Decreto 123/2014, que regula el Reglamento de la Ley 14/2011 y al quedar transformada en una sociedad limitada, también tendremos que contar con el Real Decreto Legislativo 1/2010, de 2 de julio, por el que se aprueba el texto refundido de la Ley de Sociedades de Capital y la Ley 3/2009, de 3 de abril, sobre Modificaciones Estructurales de las Sociedades Mercantiles.

Antes de entrar en la presentación del caso práctico, vamos a destacar determinados aspectos técnicos que servirán para su realización, así como para el cálculo del Fondo de Reserva Obligatorio y del Fondo de Formación y Sostenibilidad a reintegrar. El artículo 64 de la Ley 14/2011, define la duración del ejercicio económico, indicando en su apartado primero: "1. El ejercicio económico tendrá una duración de doce meses, salvo en los casos de constitución, extinción o mutaciones estructurales de la sociedad cooperativa, y coincidirá con el año natural, a menos que los estatutos dispongan lo contrario". Por su parte, el apartado segundo del mismo artículo, indica expresamente que una vez finalizado el ejercicio económico se realizará la distribución del resultado positivo o la imputación de pérdidas.

\subsection{Caso práctico: Sociedad MAPI, S.C.A. ${ }^{5}$}

La Sociedad MAPI, S.C.A., dedicada a la comercialización de productos terminados del barro, en la provincia de Córdoba, está planteándose su transformación en una sociedad limitada. Para ello, se ampara en la Ley 14/2011, de 23 de diciembre, de Sociedades Cooperativas Andaluzas, que prevé esta posibilidad en su artículo 78, en el artículo 65 del Decreto 123/2014, de 2 de septiembre por el que se aprueba su Reglamento y en la Ley 3/2009, de 3 de abril, sobre Modificaciones Estructurales de las Sociedades Mercantiles. Se presenta a continuación, en la Tabla $n^{\circ} 1$, el Balance a 31 de diciembre del año 2015, y al día anterior al acuerdo de transformación en el ejercicio 2016, al igual que la Cuenta de Pérdidas y Ganancias de 2015 y al día anterior al acuerdo de transformación (Tabla n 2).

\footnotetext{
5 El nombre de la sociedad del caso práctico es figurado para no revelar su identidad, no obstante, los datos son
} reales 
Tabla. 1. Balance de Situación a 31 de diciembre de 2015 y 15 de mayo de 2016 de MAPI, S.C.A

\begin{tabular}{|c|c|c|c|c|}
\hline & \multirow[b]{2}{*}{ ACTIVO } & \multirow{2}{*}{$\begin{array}{c}\text { Notas de la } \\
\text { Memoria } \\
\end{array}$} & \multirow{2}{*}{$\begin{array}{c}\text { Ejercicio } \\
2015 \\
\end{array}$} & \multirow{2}{*}{$\begin{array}{c}\text { Ejercicio } \\
2016 \\
\end{array}$} \\
\hline & & & & \\
\hline A) & ACTIVO NO CORRIENTE & & $\mathbf{1 . 6 8 0 . 6 4 0 , 0 0}$ & $1.693 .092,21$ \\
\hline I. & Inmovilizado intangible & Nota 5 & $100.000,00$ & $94.300,00$ \\
\hline 3. & Patentes, licencias, marcas y similares & & $80.000,00$ & $76.500,00$ \\
\hline 5. & Aplicaciones informáticas & & $20.000,00$ & $17.800,00$ \\
\hline II. & Inmovilizado material & Nota 6 & $1.330 .640,00$ & $1.309 .922,71$ \\
\hline 1. & Terrenos y construcciones & & $980.500,00$ & $974.371,88$ \\
\hline 2. & Instalaciones técnicas y otro inmovilizado material & & $350.140,00$ & $335.550,83$ \\
\hline III. & Inversiones Inmobiliarias & Nota 7 & $250.000,00$ & $250.000,00$ \\
\hline 1. & Terrenos & & $250.000,00$ & $250.000,00$ \\
\hline VI. & Activo por impuesto diferido & & $\mathbf{0 , 0 0}$ & $\mathbf{3 8 . 8 6 9 , 5 0}$ \\
\hline B) & ACTIVO CORRIENTE & & $\mathbf{3 7 5 . 3 8 7 , 4 1}$ & $281.882,41$ \\
\hline II. & Existencias & Nota 9 & $39.000,00$ & $31.400,00$ \\
\hline 1. & Comerciales & & $34.500,00$ & $31.400,00$ \\
\hline 6. & Anticipos a proveedores & & $4.500,00$ & 0,00 \\
\hline III. & Deudores comerciales y otras cuentas a cobrar & & $166.600,00$ & $164.900,00$ \\
\hline 1. & Clientes por ventas y prestaciones de servicios & Nota 10 & $125.400,00$ & $122.300,00$ \\
\hline 3. & Deudores varios & Nota 10.1 & $25.400,00$ & $26.800,00$ \\
\hline 6. & Otros créditos con las Administraciones Públicas & Nota 11 & $15.800,00$ & $15.800,00$ \\
\hline v. & Inversiones financieras a corto plazo & Nota 13 & $30.000,00$ & $31.000,00$ \\
\hline 1. & Instrumentos de patrimonio & & $26.500,00$ & $27.500,00$ \\
\hline 2. & Créditos a empresas & & $3.500,00$ & $3.500,00$ \\
\hline VI. & Periodificaciones a corto plazo & & $6.042,41$ & $6.042,41$ \\
\hline VII. & Efectivo y otros activos líquidos equivalentes & Nota 14 & $133.745,00$ & $48.540,00$ \\
\hline 1. & Tesorería & & $98.745,00$ & $38.540,00$ \\
\hline \multirow[t]{2}{*}{2.} & Otros activos liquidos equivalentes & & $35.000,00$ & $10.000,00$ \\
\hline & TOTAL ACTIVO $($ A + B) & & $2.056 .027,41$ & $1.974 .974,62$ \\
\hline
\end{tabular}

\begin{tabular}{|c|c|c|c|c|}
\hline & \multirow[b]{2}{*}{ PATRIMONIO NETO + PASIVO } & \multirow{2}{*}{$\begin{array}{c}\text { Notas de la } \\
\text { Memoria }\end{array}$} & \multirow{2}{*}{$\begin{array}{c}\text { Ejercicio } \\
2015 \\
\end{array}$} & \multirow{2}{*}{$\begin{array}{c}\text { Ejercicio } \\
2016\end{array}$} \\
\hline & & & & \\
\hline A) & PATRIMONIO NETO & & $412.385,44$ & $254.298,14$ \\
\hline A-1) & Fondos propios & Nota 10.1 & $346.985,44$ & $191.623,14$ \\
\hline I. & Capital. & & $250.000,00$ & $259.329,94$ \\
\hline 1. & Capital suscrito cooperativo. & & $250.000,00$ & $259.329,94$ \\
\hline II. & Reservas & & $85.000,00$ & $\mathbf{8 7 . 7 7 1 , 2 0}$ \\
\hline 1. & Fondo de reserva obligatorio. & & $85.000,00$ & $87.771,20$ \\
\hline V. & Excedente de la cooperativa (positivo o negativo) & Nota 3 & $11.985,44$ & $-155.478,00$ \\
\hline A-3) & Subvenciones, donaciones y legados recibidos & Nota 10.2 & $65.400,00$ & $62.675,00$ \\
\hline B) & PASIVO NO CORRIENTE & & 912.508,73 & $854.263,15$ \\
\hline I. & Fondo de educación, formación y sostenibilidad & Nota 11 & $1.292,07$ & 599,27 \\
\hline IV. & Deudas a largo plazo & Nota 8.1 & $903.950,00$ & $846.700,00$ \\
\hline 1. & Deuda con entidades de crédito & & $878.450,00$ & $823.500,00$ \\
\hline 2. & Acreedores por arrendamiento financiero & Nota 7 & $25.500,00$ & $23.200,00$ \\
\hline VI. & Pasivos por impuesto diferido & Nota 12.2 & $7.266,66$ & $6.963,88$ \\
\hline C) & PASIVO CORRIENTE & & $731.133,24$ & $866.413,33$ \\
\hline V. & Deudas a corto plazo & Nota 8.1 & $164.289,00$ & $132.200,00$ \\
\hline 1. & Deudas con entidades de crédito & & $154.789,00$ & $125.800,00$ \\
\hline 2. & Acreedores por arrendamiento financiero & Nota 7 & $9.500,00$ & $6.400,00$ \\
\hline VII. & Acreedores comerciales y otras cuentas a pagar & Nota 8.1 & $566.844,24$ & $734.213,33$ \\
\hline 1. & Proveedores & Nota 8.1 & $116.737,41$ & $222.956,45$ \\
\hline 2. & Acreedores varios & Nota 8.1 & $147.500,00$ & $224.319,50$ \\
\hline 4. & Personal (remuneraciones pendientes de pago) & Nota 8.1 & $170.500,00$ & $184.500,00$ \\
\hline 6. & Otras deudas con las Administraciones Públicas & Nota 12.1 & $132.106,83$ & $102.437,38$ \\
\hline & TOTAL PATRIMONIO NETO Y PASIVO (A+B+C) & & $2.056 .027,41$ & $1.974 .974,62$ \\
\hline
\end{tabular}

Fuente: Elaboración propia 
Tabla. 2. Cuenta de Pérdidas y Ganancias correspondientes a los ejercicios a 31 de diciembre de 2015 y 15 de mayo de 2016 de MAPI, S.C.A

\begin{tabular}{|c|c|c|c|}
\hline & & (DEBE) HABER & \begin{tabular}{|c|} 
(DEBE) HABER \\
2016
\end{tabular} \\
\hline 1. & Importe neto de la cifra de negocios & $2.350 .111,12$ & $2.458 .715,00$ \\
\hline 2. & Variación de existencias de productos terminados y en curso de fabricación & 0,00 & 0,00 \\
\hline 3. & Trabajos realizados por la cooperativa para su activo & 0,00 & 0,00 \\
\hline 4. & Aprovisionamientos & $-480.154,12$ & $-502.154,12$ \\
\hline a) & Consumos de existencias & $-480.154,12$ & $-502.154,12$ \\
\hline b) & Otros aprovisionamientos & 0,00 & 0,00 \\
\hline 5. & Otros ingresos de explotación & 0,00 & 0,00 \\
\hline a) & ingresos por operaciones con socios & 0,00 & 0,00 \\
\hline b) & Otros ingresos & 0,00 & 0,00 \\
\hline 6. & Gastos de personal & $-1.360 .959,77$ & $-1.548 .631,62$ \\
\hline a) & Servicios de trabajo de socios & $-1.360 .959,77$ & $-1.548 .631,62$ \\
\hline b) & Otros gastos de personal & 0,00 & 0,00 \\
\hline 7. & Otros gastos de explotacion & $-353.789,60$ & $-456.140,00$ \\
\hline 8. & Amortización del inmovilizado & $-75.835,12$ & $-76.135,64$ \\
\hline 9. & Imputación de subvenciones de inmovilizado no financiero y otras & $5.450,00$ & $5.450,00$ \\
\hline 10. & Excesos de provisiones & 0,00 & 0,00 \\
\hline 11. & Deterioro y resultado por enajenaciones del inmovilizado & 0,00 & 0,00 \\
\hline 12. & Fondo de Educación, Formación y Promoción & 692,80 & 0,00 \\
\hline a) & Dotación & 692,80 & 0,00 \\
\hline b) & Subvenciones, donanciones y ayudas y sanciones & 0,00 & 0,00 \\
\hline A) & RESULTADO DE EXPLOTACIÓN $(1+2+3+4+5+6+7+8+9+10+11+12)$ & $85.515,31$ & $-118.896,38$ \\
\hline 13. & Ingresos financieros & 0,00 & 0,00 \\
\hline a) & De socios & 0,00 & 0,00 \\
\hline b) & Otros & 0,00 & 0,00 \\
\hline 14. & Gastos financieros & $-72.352,11$ & $-75.451,12$ \\
\hline & Intereses y retorno obligatorio de las aportaciones al capital social y de otros & & \\
\hline a) & fondos calificados con características de deuda & 0,00 & 0,00 \\
\hline b) & Otros gastos financieros & $-72.352,11$ & $-75.451,12$ \\
\hline 15. & Variación de valor razonable en instrumentos financieros & 0,00 & 0,00 \\
\hline 16. & Diferencias de cambio & 0,00 & 0,00 \\
\hline 17. & Deterioro y resultado por enajenaciones de & 0,00 & 0,00 \\
\hline B) & RESULTADO FINANCIERO $(13+14+15+16+17)$ & $-72.352,11$ & $-75.451,12$ \\
\hline C) & RESULTADO ANTES DE IMPUESTOS (A+B) & $13.163,20$ & $-194.347,50$ \\
\hline 18. & Impuestos sobre beneficios & $-1.177,76$ & $38.869,50$ \\
\hline E) & RESULTADO DEL EJERCICIO $(\mathrm{C}+18)$ & $11.985,44$ & $-155.478,00$ \\
\hline
\end{tabular}

Fuente: Elaboración propia

\subsection{Comentarios a los estados económico-financieros}

A 31 de diciembre de 2015 existen en el balance algunos datos que resulta importante destacar, los cuales utilizaremos posteriormente para realizar el cálculo de los fondos a reintegrar en el proceso de transformación de la cooperativa. Por un lado, el Fondo de Reserva Obligatorio que presenta la cooperativa tiene un saldo de 85.000 euros; el Fondo de educación, formación y sostenibilidad es de 1.292,07 euros, así como el Resultado después de Impuestos, denominado Excedente de la cooperativa, de 11.985,44 euros. Para llegar a dicho excedente, se ha tenido que realizar un proceso de cálculo, ya que previo al impuesto, se debe computar la dotación de fondos sociales cooperativos. El artículo 68 de la Ley 14/2011, establece en su apartado 2 que, en todo caso, habrán de dotarse los fondos sociales obligatorios, una vez deducidas las pérdidas de ejercicios anteriores y antes de la consideración del impuesto de sociedades. Para ello, vamos a considerar a efectos del cálculo del citado impuesto sobre sociedades, que los gastos e ingresos 
ocurridos en 2015 y 2016 son imputables fiscalmente al período en el que se realizan (años 2015 y 2016), al igual que todos los gastos en los que incurre la sociedad son deducibles en el mismo año. Asimismo, vamos a tener en cuenta que todos los ingresos y gastos tienen naturaleza cooperativa y que la entidad cumple con todos los requisitos previstos legalmente para ser una cooperativa especialmente protegida. Ahora vamos a considerar que no existen diferencias permanentes ni temporarias, a efectos de liquidación del impuesto sobre sociedades, salvo el 50\% del Fondo de Reserva Obligatorio (FRO), que se considera como un gasto fiscal deducible y que se tiene que ajustar al calcular el impuesto sobre sociedades.

Para obtener el excedente positivo de 2015, que figura en el balance y en la cuenta de pérdidas y ganancias, debemos partir del resultado antes de impuestos y fondos 13.856,00 euros, cuyo cálculo se explica en el siguiente epígrafe 2.3. Al no existir pérdidas de ejercicios anteriores que restar, procederíamos a dotar el Fondo de Reserva Obligatorio en un porcentaje del 20\% de dicho resultado, 2.771,20 euros; así como un Fondo de educación, formación y sostenibilidad (FEFS) del 5\%, 692,80 euros. Para determinar los porcentajes que hemos tomado para la dotación de los fondos, nos tenemos que remitir al artículo 68 de la Ley 14/2011. En su apartado $2 \mathrm{a}$, señala que los resultados cooperativos, se destinarán, como mínimo, un $20 \%$ al FRO, hasta que este alcance el $50 \%$ del capital social. En nuestro caso práctico, el capital social es de 250.000 euros y el FRO de 85.000 euros, por lo que se dotará como mínimo el $20 \%$. Continúa el apartado 2 del citado artículo, y menciona que se dotará al menos un 5\% al FEFS.

Una vez dotados los fondos, debemos contabilizar como gasto la dotación al FEFS y reflejarlo en la cuenta de pérdidas y ganancias como un gasto, y, en el pasivo del balance, como una obligación. Dicho criterio aparece recogido en la norma $6^{\mathrm{a}}$ de la Orden EHA/3360/2010 de 21 de diciembre, por la que se aprueban las normas sobre aspectos contables de las cooperativas y en la Consulta 3 del Boletín Oficial del Instituto de Contabilidad y Auditoría de Cuentas (BOICAC) $\mathrm{n}^{\circ} 76$. En el caso práctico que nos ocupa, dicho importe se encuentra reflejado en el pasivo corriente por importe total de 1.292,07 euros, cantidad que se obtiene de sumar la dotación al FEFS del ejercicio 2015 (692,80 euros), con cantidades que ya tenía en contabilidad la sociedad de dotaciones de ejercicios anteriores.

Por último, indicar que no aparece reflejado el crédito impositivo del impuesto de sociedades del ejercicio 2016 en el balance de situación, al entender la empresa, de acuerdo con la Consulta 10 del Instituto de Contabilidad y Auditoría de Cuentas $80 / 2009$, que no era probable que obtuviera beneficios para compensar dichas pérdidas en un periodo máximo de diez años.

\subsection{Cálculo del Impuesto de Sociedades}

Para calcular el impuesto sobre sociedades que se devenga en la cooperativa tendremos que realizar los siguientes cálculos presentados en la tabla siguiente: 
Tabla. 3. Cálculo del Resultado del Ejercicio después de impuestos

\begin{tabular}{|l|r|}
\hline Resultado antes de impuestos y fondos & $13.856,00$ \\
\hline - Dotación al Fondo de Reserva Obligatorio (FRO), 20\% & $2.771,20$ \\
\hline - Dotación al Fondo de Educación Formación y Sostenibilidad (FEFS), 5\% & 692,80 \\
\hline Resultado antes de impuestos y después de restar FEFS & $13.163,20$ \\
\hline - Diferencia Permanente: 50\% FRO & $1.385,60$ \\
\hline Base Imponible & $11.777,60$ \\
\hline Tipo gravamen cooperativo & $20,00 \%$ \\
\hline Cuota & $2.355,52$ \\
\hline Bonificación cooperativas especialmente protegidas, 50\% & $1.177,76$ \\
\hline Resultado del ejercicio después de fondos e impuestos & $11.985,44$ \\
\hline
\end{tabular}

Fuente: Elaboración propia

Partiendo del resultado antes de impuestos y fondos, 13.856,00 euros, y una vez calculados los fondos, tenemos que restar el FEFS, al ser considerado como un gasto contable, llegando al resultado antes de impuestos y después de restar el FEFS 13.163,20 euros. Para calcular el impuesto a pagar a la Agencia Estatal de Administración del Estado (AEAT) por la cooperativa, debemos indicar que la Ley 20/1990 reconoce como gasto fiscalmente deducible el 50\% de la dotación obligatoria al FRO, por lo que restamos la mitad de 2.771,20/2 = 1.385,60, obtenido de esta forma la base imponible del impuesto de sociedades. El tipo de gravamen sería del 20\%,para los resultados cooperativos, al ser las cooperativas de trabajo asociado, cooperativas especialmente protegidas(suponemos que cumple los requisitos previstos en los artículos 7, 8 y 13 de Ley 20/1990 de 19 de diciembre, sobre régimen fiscal de las cooperativas), se benefician de una bonificación del 50\% de la cuota del impuesto, por lo que el impuesto a pagar y en este caso devengado, sería de 1.177,76 euros, que restado a los 13.163,20 euros, nos daría el excedente cooperativo que aparece en balance y en la cuenta de pérdidas y ganancias de $11.985,44$ euros.

Una vez determinado el proceso de cálculo del excedente cooperativo que refleja el balance de 2015, proseguiremos con el proceso de transformación. Así, el órgano de administración de la cooperativa, deberá preparar una serie de documentación que deberá estar a disposición del socio antes de la Asamblea donde se deliberará sobre el acuerdo de transformación. En concreto:

a) El informe del órgano de administración ${ }^{6}$ que explique y justifique los aspectos jurídicos y económicos de la transformación e indique las consecuencias del mismo para las personas socias, así como su eventual impacto de género en los órganos de administración e incidencia, en su caso, en la responsabilidad social de la empresa.

b) El balance de la sociedad cooperativa que se transforma, cerrado dentro de los 8 meses anteriores a la fecha prevista para la Asamblea. (en nuestro caso práctico, sería el cerrado a 31 de diciembre de 2015). 
c) El informe de la auditoría externa de cuentas sobre el balance presentado, dado que la sociedad que se transforma está obligada a someter sus cuentas a auditoría.

d) Proyecto de escritura o estatutos de la sociedad que resulte de la transformación, así como, en su caso, otros pactos sociales que vayan a constar en documento público.

Por otro lado, el órgano de administración está obligado a informar a la Asamblea General a la que se someta la aprobación de la transformación sobre cualquier modificación importante del activo o del pasivo acaecida entre la fecha del informe justificativo de la transformación y del balance puesto a disposición de las personas socias y la fecha de la celebración de la Asamblea ${ }^{7}$. Por otra parte, una vez convocada la Asamblea de la sociedad cooperativa, el procedimiento continuará de la siguiente forma:

1. Acuerdo expreso de la Asamblea General adoptado conforme a lo establecido para las modificaciones estatutarias.

2. Publicación del acuerdo de la Asamblea General en la sede electrónica de la Consejería competente en materia de sociedades cooperativas o notificación individual a socios y acreedores. Debe transcurrir un mes como mínimo desde la fecha de publicación o desde la última notificación individual practicada para poder realizar la transformación.

3. Elevación del acuerdo a escritura pública, que contendrá todas las menciones exigidas legalmente para la constitución de la nueva entidad respetando lo dispuesto en la Ley 14/2011 y en su Reglamento.

4. El certificado del acta de la Asamblea General, o, en su caso, la escritura pública, deberá presentarse en el Registro de Cooperativas Andaluzas para inscribir la transformación correspondiente. La misma deberá ir acompañada del balance de situación cerrado el día anterior al del acuerdo de transformación, o bien el del último ejercicio si hubiesen transcurrido menos de seis meses desde el cierre del mismo y hubiese sido depositado en el domicilio social.

5. Asimismo, se deberá garantizar, en todo caso, el derecho de las personas socias a separación, cuando concurran las circunstancias establecidas en el artículo 23.3, letras b) y c) de la LGC 14/2011, de conformidad con lo establecido en el artículo 78 de la citada Ley.

\subsection{Determinación y puesta a disposición de la Administración de los fondos reintegrables}

Un último aspecto a considerar en el proceso de transformación es la determinación y puesta a disposición de la Administración de los Fondos reintegrables, tanto el FRO como el FEFS. Al respecto, el artículo 141 del Real Decreto 123/2014 establece: "El Registro de Cooperativas, una vez recabada la documentación a la que alude el artículo 65.5.d), procederá a calificarla conforme a la tramitación general establecida en la Sección $2^{a}$ de este Capítulo, con especial observancia de 
las normas relativas al activo y fondos reintegrables a la Administración”. En lo que concierne a los fondos a reintegrar a la Administración autonómica, debemos considerar varios aspectos que pueden resultar de importancia a fin de determinar los citados fondos y el momento del cálculo.

\section{Subvenciones de capital}

Una cuestión de especial relevancia para el caso de transformación de una cooperativa son las subvenciones de capital, en cuanto que no existe una única interpretación. Según algunos autores "parece claro que, en tanto no se pierda el derecho a la subvención como consecuencia de la operación de transformación, el ingreso diferido terminará convirtiéndose en beneficio, y, por tanto, parece coherente desde un punto de vista económico-financiero, asignar la parte del mismo a la Administración".

Desde nuestro punto de vista, no podemos estar de acuerdo con este planteamiento seguido por algunos autores por los razonamientos que a continuación se exponen.

Primero, debemos distinguir si las subvenciones de capital que la cooperativa tiene reflejadas dentro de su patrimonio neto, resultarán o no reintegrables, a consecuencia del proceso de transformación, ya que, en tal caso, deberíamos de reclasificarlas en el pasivo de la sociedad. Además, se habría de analizar si la Administración nos solicita el reintegro de lo pendiente de imputar o del total de la subvención. En el caso que nos soliciten la devolución de lo pendiente de imputar, que aparecerá recogido en la cuenta 130 Subvenciones de capital y 479 Pasivo por diferencias temporarias imponibles, tan sólo tendríamos que realizar una reclasificación de dichas partidas dándolas de baja y apareciendo en el pasivo una obligación de devolución por subvenciones reintegrables. Si nos pidieran la totalidad, el proceso se complicaría, puesto que como ya tendríamos parte de la subvención imputada en la cuenta de pérdidas y ganancias de los ejercicios anteriores, al tener que reintegrarlas ahora, deberíamos reflejar una pérdida en nuestra cuenta de pérdidas y ganancias, que sería un resultado cooperativo, al igual que fue ingreso cooperativo cuando se imputó. Cabría plantearse si dicho gasto se debería reflejar en pérdidas y ganancias del ejercicio, o al corresponder a ejercicios anteriores iría contra reservas. A nuestro juicio, al ser un gasto surgido en este ejercicio, por dejar de cumplir los requisitos para mantener la subvención, se entiende que debería aplicarse como gasto del ejercicio en cuestión.

Si la subvención no quedara afecta por el proceso de transformación, y, por tanto, no tuviera que ser reintegrada, se plantearía la duda, como dice el autor indicado anteriormente, de si dichos ingresos deben ser considerados a la hora de calcular el importe del Fondo de Reserva Obligatorio, "ya que son ingresos que terminarán incorporándose a la cuenta de resultados de la sociedad y ya están recogidos contablemente."

8 Rojo Ramírez, A.; Esteban Cerdán, A.; Sánchez Pérez, J.A. (2001), La transformación de cooperativas. Especial referencia al caso andaluz. Ciriec-España, Revista de Economía Pública, Social y Cooperativa, $\mathrm{N}^{\circ}$ 38, pp. 57-76. 
En este punto, tal y como ya hemos indicado más arriba, no podemos estar de acuerdo con lo manifestado. El motivo es que dichas subvenciones reconocidas, lo estarán normalmente al tener la consideración de subvenciones de capital al haber realizado la cooperativa inversiones en inmovilizado, el cual habrá sido financiado en parte por dicha subvención. La amortización anual de dicho inmovilizado se incorporará a la cuenta de pérdidas y ganancias. Por tanto, si se tuvieran en cuenta los ingresos a imputar en un futuro por subvenciones concedidas a la fecha de la transformación, a efectos de determinar el Fondo de Reserva Obligatorio, también se debería considerar dicho gasto de amortización, razonamiento muy lógico por el criterio de correlación de ingresos y gastos, que sigue inspirando el plan contable, a pesar de que haya sido eliminado como principio contable.

En resumen, no parece lógico que se considere el ingreso, y no así el gasto, por lo que abogamos que ambos conceptos sean reconocidos en las cuentas de pérdidas y ganancias de cada ejercicio en función de su imputación. De esta manera, no sería correcto afectar al cálculo del Fondo de Reserva Obligatorio a efectos de su transformación, ya que en el momento en que se produzca el cambio a la nueva forma jurídica, tanto los ingresos generados por dicha subvención, como los gastos por amortización de la inversión, no tendrán la consideración de cooperativos.

\section{Momento y cálculo de los fondos reintegrables a la Administración}

Respecto al momento y a la forma en la que se debe calcular el valor de los fondos, existe cierta confusión, dado que ninguna normativa lo especifica expresamente, lo cual da lugar a bastantes problemas con la Administración en la práctica profesional. Centrándonos en el caso planteado, se ha producido un acuerdo de transformación con fecha 16 de mayo de 2016. De acuerdo con el artículo 64 de la Ley 14/2011, se ha producido una modificación estructural de la cooperativa, de las recogidas en el Capítulo VII de la Ley de Cooperativas Andaluzas, con el nombre de Modificaciones Estatutarias y Estructurales y regulada en el artículo 78 de la citada ley.

A efectos de calcular los fondos sociales a reintegrar a la Administración, tendremos que determinar en qué momento la sociedad deja de ser cooperativa y pasa a constituirse, en nuestro caso, en sociedad limitada. Según establece el citado artículo 64.1 de la Ley de Cooperativas Andaluza, el ejercicio económico tendrá una duración de doce meses, salvo en los casos de constitución, extinción o mutaciones estructurales de la sociedad cooperativa, y coincidirá con el año natural, a menos que los estatutos dispongan lo contrario. En el caso que nos ocupa se ha producido una mutación estructural, y, por tanto, será ese el momento en el que haya que determinar el importe de los fondos, ya que a partir de ahí los ingresos y gastos dejarán de ser cooperativos. La problemática se suscita a la hora de determinar dicho momento.

Por un lado, tenemos que la Ley 3/2009 de Modificaciones Estructurales señala en su artículo 19, que la eficacia de la transformación se producirá con la inscripción de la escritura pública en el Registro Mercantil. En este sentido, el artículo 116 del Real Decreto 123/2014, referente al carácter y eficacia de las inscripciones, señala que "la inscripción en el Registro de Cooperativas Andaluzas tendrá carácter obligatorio en relación con aquellos actos en que la Ley 14/2011, 
de 23 de diciembre, y el presente Reglamento así lo prevean, teniendo carácter constitutivo los previstos en el artículo 119.1 de la citada Ley, y declarativo, todos los demás". Asimismo, menciona que "Los actos cuya inscripción registral tenga carácter constitutivo no podrán ser aplicados válidamente hasta tanto no se practique aquella."

El artículo 119.1 de la Ley 14/2001 señala que la inscripción de los actos de constitución, modificación de estatutos sociales, fusión, escisión, transmisión, cesión global del activo y del pasivo, transformación, disolución y reactivación de las sociedades cooperativas tendrá eficacia constitutiva. Luego, parece algo pacífico que la inscripción en el Registro de las operaciones estructurales tenga carácter constitutivo, al configurar alteraciones morfológicas de las sociedades, equiparándolas, por tanto, a la constitución. Lo que ya no parece tan pacífico es cuándo se entiende realizado dicho acto, si cuando se produce la inscripción definitiva, o cuando se produce el asiento de entrada registral.

Al respecto, el artículo 55.1 del Reglamento del Registro Mercantil, señala que se considera como fecha de la inscripción la fecha del asiento de presentación. En este sentido, existen posturas contrapuestas que señalan que los efectos se producen con la inscripción y no con la presentación. Existen dos resoluciones de la Dirección General de Registros y Notariado, que defienden esta postura del 20 de septiembre y del 13 de octubre 2011 y una sentencia de la Audiencia Provincial de Pontevedra 466/2011, de 19 de septiembre, que en relación a los efectos de una transformación de cooperativa en sociedad limitada, viene a señalar que, hasta que no se produzca la inscripción en el Registro mercantil de la transformación, no se produce el cambio de régimen jurídico.

No obstante, entendemos que el Tribunal Supremo, en su Sentencia STS, 2a, 21.5.2012, ha dejado claro este aspecto, al afirmar la vigencia del citado artículo 55.1 RRM, estableciendo como tiempo de acceso al Registro el del asiento de presentación, datando en esta fecha la posterior inscripción. Por tanto, considerando dicha situación, entendemos que el momento en el que se deberían de calcular los fondos a reintegrar sería la fecha del asiento de presentación en el Registro. Aunque aquí volvería a surgir la duda, si en el Registro de Cooperativas o en el Mercantil, ya que primero se presentarán en el Registro de Cooperativas y una vez inscrita esta baja, se presentará la documentación en el Registro Mercantil. Entendemos que debería ser la fecha de presentación en el Registro de Cooperativas, a pesar de que el artículo 55.1 RRM, haga referencia al Registro Mercantil, ya que en caso de entenderse así, se produciría una situación insalvable, puesto que para poder realizar la inscripción en el Registro Mercantil es necesario tener previamente la baja del Registro de Cooperativas y esta no se producirá mientras no se haya realizado el ingreso de los fondos a reintegrar. Por ello, entendemos que en el caso de la transformación de una cooperativa en sociedad limitada, el momento viene determinado por la fecha de presentación en el Registro de Cooperativas.

Una vez definido el momento en el que se tienen que determinar los fondos a reintegrar, se plantea otro problema a la hora de efectuar el asiento de la documentación en el Registro de Cooperativas, pues la cooperativa ya debería aportar, junto con el resto de documentación citada, los ingresos de los fondos en el modelo 046; incluso, entendemos que en la Asamblea de aprobación de la 
transformación, el Consejo Rector de la cooperativa ya ha debido informar a los socios sobre las cuantías a reintegrar. Si tenemos en cuenta lo indicado en el artículo 64.1 de la Ley 14/2011, citado anteriormente, podemos considerar como fecha para la determinación de los fondos, el acuerdo de transformación, debiendo proponer una distribución de los resultados a efectos de la determinación de los importes destinados al FRO y al FEFS.

En nuestro ejemplo, el cálculo del fondo a ingresar a la Administración lo realizaríamos en base al balance formulado al día anterior al acuerdo, es decir, el 15 de mayo de 2016.

Tabla. 4. Cálculo del Fondo de Reserva Obligatorio a Reintegrar

\begin{tabular}{|l|r|}
\hline Fondo Reserva Obligatorio (FRO) & $85.000,00$ \\
\hline Dotación del FRO del Ejercicio & $2.771,20$ \\
\hline Dotación 50\% de las Pérdidas del Ejercicio 2015 & $-77.739,00$ \\
\hline Fondo de Reserva Obligatorio & $10.032,20$ \\
\hline Fondo a Reintegrar 50\% & $\mathbf{5 . 0 1 6 , 1 0}$ \\
\hline
\end{tabular}

Fuente: Elaboración propia

En los estatutos sociales deberá de recogerse la distribución del 50\% de FRO a los socios a efectos de reintegrar a la Administración el otro 50\%, en caso de que no se recoja deberá de reintegrarse el $100 \%$ a la Administración. Respecto al importe del FEFS a ingresar a la Administración, será el importe total que figure en el balance al día del acuerdo; en nuestro caso, 599,27 euros, ya que durante el ejercicio 2016, al haber existido pérdidas, no se corresponde imputar cantidad alguna y la diferencia con los existentes al 31/12/2015 son aplicaciones de dicho fondo realizados en 2016 y que posiblemente la Administración nos pida justificar. No obstante, debemos considerar que desde la fecha de la aprobación en Asamblea, hasta que la documentación se pueda presentar en el Registro de Cooperativas, va a mediar como mínimo un periodo de un mes, en el que deberá estar publicado el acuerdo, por lo que entendemos que si durante dicho periodo se hubieran producido operaciones que hayan generado beneficios o pérdidas, se debería realizar un nuevo cálculo de los fondos a efectos de hacer el ingreso lo más ajustado posible a la fecha del asiento de presentación.

\section{Fiscalidad del proceso de transformación}

Especial mención requiere el impuesto de sociedades (IS) en el caso de la transformación. El artículo 28 de la ley 27/2014, de 27 de noviembre del impuesto sobre sociedades, referente al periodo impositivo, indica que dicho periodo coincidirá con el ejercicio económico de la sociedad, salvo cuando concurran una serie de requisitos, entre ellos el descrito en el apartado d) siguiente: “Cuando se produzca la transformación de la forma societaria de la entidad, o la modificación de su estatuto o de su régimen jurídico, y ello determine la modificación de su tipo 
de gravamen o la aplicación de un régimen tributario distinto. La renta derivada de la transmisión posterior de los elementos patrimoniales existentes en el momento de la transformación o modificación, se entenderá generada de forma lineal, salvo prueba en contrario, durante todo el tiempo de tenencia del elemento transmitido. La parte de dicha renta generada hasta el momento de la transformación o modificación se gravará aplicando el tipo de gravamen y el régimen tributario que hubiera correspondido a la entidad de haber conservado su forma, estatuto o régimen originario."

En este contexto, podemos encontrarnos con diferentes situaciones según lo establecido en este artículo:

Primera.- La sociedad cooperativa transformada es especialmente protegida o protegida, y pasa a ser sociedad limitada; en este caso, se estaría produciendo un cambio de régimen tributario y del tipo de gravamen aplicable. El primero, al pasar de tributar de un régimen especial, como sería el de cooperativa protegida o especialmente protegida, a otro régimen distinto. El segundo, porque se produciría un cambio de tipo de gravamen, en tanto que, tal y como ya hemos comentado, el régimen cooperativo tributa a un tipo distinto al general del impuesto.

Segunda.- Que la cooperativa transformada no cumpla los requisitos previstos para ser considerada como especialmente protegida o protegida y tributara por el régimen general y al tipo general previsto, con lo que no se produciría alteración alguna de las previstas en el artículo 28 de la Ley 27/2014.

En el primero de los casos, tendríamos dos periodos impositivos, uno, que concluiría con la inscripción de la transformación, $y$, otro, que iría desde esa fecha de la inscripción hasta el 31 de diciembre, si su ejercicio económico coincidiera con el año natural. En el segundo de los casos, al no darse ninguno de los supuestos contemplados en el citado artículo, a pesar del proceso de transformación, desde el punto de vista del impuesto sobre sociedades, no se produciría ninguna interrupción del periodo impositivo, con lo que existirá un solo impuesto sobre sociedades. Por ello, al realizar la transformación de una cooperativa en sociedad limitada, hay que prestar especial atención a la fecha exacta en la que se entiende finalizado el periodo impositivo de la cooperativa, para así poder realizar los impuestos de sociedades en cada periodo impositivo.

La consulta tributaria vinculante V2878-13 plantea una cuestión principal en cuanto al momento en que produce efectos la transformación de una cooperativa de trabajo asociado andaluza con carácter fiscalmente protegido, con arreglo a lo dispuesto en la Ley 20/1990, sobre régimen fiscal de cooperativas. En ella se plantea el dilema acerca de cuándo produce efectos la transformación: si es cuando finaliza el período impositivo de la cooperativa, o cuando se inscribe la nueva sociedad en el Registro Mercantil. A esta cuestión la DGT (Dirección General de Tributos) hace referencia al artículo 26.2 del texto refundido de la Ley del Impuesto sobre Sociedades (TRLIS), actualmente ya no vigente, siendo actualmente el 28 de la Ley 27/2014, aunque con un contenido idéntico, el cual establecía en el apartado 2 d): "Cuando se produzca la transformación de la forma jurídica de la entidad y ello determine la modificación de su tipo de gravamen o la aplicación de un régimen tributaria especial".

Mercantilmente, el artículo 19 de la Ley 3/2009, de 3 de abril, sobre Modificaciones Estructurales de las Sociedades Mercantiles, establece en relación a 
la eficacia de la transformación, que quedará supeditada a la inscripción de la escritura pública en el Registro Mercantil. No obstante, entendemos que dicho momento, de acuerdo con el 55.1 del Reglamento del Registro Mercantil (RRM), sería el del asiento de presentación en el Registro Mercantil. En el aspecto fiscal, entendemos que nos tenemos que remitir a la fecha de presentación en el Registro Mercantil, que sería la fecha a la que hace referencia el artículo 55.1 del Reglamento del RM.

Respecto a los impuestos indirectos como el impuesto sobre el valor añadido (IVA), el proceso de transformación no causa ningún problema, ya que, al no existir cambio en la persona jurídica, se continuarán presentando las declaraciones trimestrales o mensuales como si no se hubiera producido alteración alguna. Una vez realizada la inscripción las nuevas declaraciones se realizarán con los nuevos datos fiscales, sin que suponga alteración alguna en los modelos que se presentan.

En relación al impuesto de transmisiones patrimoniales (ITP), operaciones societarias y actos jurídicos documentados (AJD), la consulta vinculante de la DGT, V2396-10 concluye que la transformación de sociedad no constituye hecho imponible de la modalidad de operaciones societarias; $y$, respecto al de los actos jurídicos documentados, sólo constituye hecho imponible por la modalidad de documentos notariales, en cuanto a la cuota fija, pero no en cuanto a la variable.

\section{Efectos contables del momento de la transformación}

Desde la óptica contable se debe determinar el momento desde el que la sociedad dejará de usar las normas contables para empresas cooperativas y comenzará a usar las correspondientes al Plan General de Contabilidad (PGC) que le aplique.

El marco conceptual del PGC indica claramente que a la hora de determinar el registro contable se debe atender al fondo económico, es decir, al momento del control económico de los mismo, independientemente de la fecha de producción jurídica real. Partiendo de que en el proceso de transformación no se produce un cambio del control económico de los activos y pasivos, como puede ocurrir en otro tipo de modificaciones estructurales (como por ejemplo, en una fusión), salvando la posible entrega a la Administración pública de los Fondos a reintegrar, los efectos contables no van a ir más allá del cambio de codificación contable y del reajuste de las cuentas de fondos propios entre los socios que hayan quedado después del proceso de transformación mercantil y los que hayan decidido causar baja. Cabría plantearse cuál será la fecha que se deberá considerar a efectos de presentar las cuentas anuales de la sociedad como cooperativa o como sociedad limitada. Se entiende que se debe estar a la fecha de inscripción, y, retroactivamente, a la fecha de presentación del asiento en el Registro Mercantil. Así, si una cooperativa, a la fecha de formulación de sus cuentas anuales no ha sido inscrita en el Registro Mercantil, deberá presentar sus cuentas anuales como sociedad cooperativa hasta el 31 de diciembre, en caso de que coincida con la fecha de su cierre de ejercicio económico. Obviamente, la sociedad tendrá que informar de su proceso de transformación en la Memoria.

No obstante, aquí podría surgir el problema de que la entidad haya sido dada de baja en el Registro de Cooperativas y aún no se haya inscrito en el Registro Mercantil a la fecha de formulación de sus cuentas. En este caso, entendemos que 
las cuentas anuales se deberían elaborar de acuerdo al PGC y ser presentadas en el Registro Mercantil una vez inscrita.

Otro aspecto a considerar sería los efectos contables sobre el patrimonio neto de la cooperativa en caso de que ésta no hubiera adaptado los mismos a la normativa vigente y siguiera manteniendo en el mismo incluso los que tienen carácter de reintegrables. En este caso, la sociedad limitada deberá traspasar a pasivo aquella parte de los mismo que presenten carácter de reintegrables y mantener como patrimonio neto solo el resto.

\section{Conclusiones}

El objetivo fundamental perseguido en este artículo es establecer la forma y el momento de calcular los fondos a reintegrar a la Administración en un proceso de transformación de una sociedad cooperativa en una sociedad limitada. La práctica profesional constata la acuciante falta de una estandarización del proceso. El hecho de que el Registro de Cooperativas tenga que emitir un certificado donde se comunica la baja de la cooperativa en el mismo, para que ésta pueda ser inscrita en el Registro Mercantil, actúa como freno de muchos procesos de transformación que no se llevan a cabo, al tener que afrontar pago de cantidades de fondos sociales requeridos por la Administración sin una base objetiva. En concreto, hemos podido comprobar situaciones donde se solicita a la empresa los fondos sociales que existen en el último balance cerrado y depositado en el Registro de Cooperativas, sin tener en cuenta los resultados que han podido acaecer durante el ejercicio en el que se está aprobando la transformación. Sería oportuno que las sociedades una vez transformadas pudieran inscribirse en el Registro Mercantil, sin la necesidad de un certificado de baja como cooperativa, y, en caso de que el Registro de Cooperativas tuviera algo que exigir, lo solicitara de manera independiente y pudiera ser objeto de recurso por parte de la empresa, pero sin paralizar un proceso iniciado por decisión del órgano supremo de la cooperativa que es la Asamblea de socios.

Hemos podido analizar que no existe un consenso en relación a la fecha en la que la transformación produce efectos jurídicos. Mientras, la Dirección General de Registros y Notariado y alguna jurisprudencia de tribunales marcaba como fecha la inscripción en el Registro Mercantil, el TS vino a reafirmar la vigencia del artículo 55.1 del RMM, el cual retrotrae la fecha, una vez realizada la inscripción, a la de la presentación del asiento en el Registro Mercantil (RM).No obstante, a efectos de calcular el importe de los fondos a reintegrar a la Administración en caso de transformación de cooperativas, no puede quedarse en dicha fecha, ya que para poder llegar a la fecha de presentación en el RM, antes se ha debido obtener la certificación de baja del Registro de Cooperativas y para ello se tendría que haber reintegrado los fondos. Por tanto, entendemos que la fecha en la que se debe llevar a cabo el cálculo definitivo de los fondos sería la de la presentación en el Registro de Cooperativas, por analogía a lo establecido en el artículo 55.1 del RM.

Será ese el momento en el que se deberán de calcular de manera definitiva los ingresos y los gastos cooperativos, así como determinar el excedente obtenido desde el inicio del ejercicio económico hasta esa fecha, donde se concluye el ejercicio al producirse una modificación estructural. El resultado obtenido deberá 
distribuirse de acuerdo con la normativa de cooperativas, de manera que si el excedente es positivo, se deberán dotar los fondos oportunos, incrementado los existentes a ese momento; y, si existen pérdidas, se podría reducir el FRO en función del destino que se otorguen a las mismas.

En el plano fiscal, en el impuesto de sociedades tendremos que estar pendientes del régimen de tributación de la cooperativa, de manera que si tributa como cooperativa protegida o especialmente protegida se producirán dos periodos de devengo, uno, que irá desde el inicio del periodo hasta la inscripción de la transformación, con retroacción de la fecha a la del asiento de presentación en el Registro Mercantil, y, otro, desde ese momento hasta la conclusión del ejercicio.

Desde el punto de vista de la imposición indirecta, el proceso de transformación tiene poca repercusión, el IVA no se verá afectado, continuando la empresa presentando sus declaraciones de manera periódica, aunque se haya producido la modificación del nombre y el CIF, del cual solo variará su letra. Por su parte, el impuesto de transmisiones patrimoniales y actos jurídicos documentados tampoco tendrá protagonismo; en este sentido, tributará la operación sólo por actos jurídicos documentados en su versión de documentos notariales cuota fija.

En el plano de la contabilidad, el proceso de transformación tendrá efectos en la modificación del plan contable que afecta, dejando de ser aplicable la norma contable de cooperativas y aplicándose el PGC.

Sí habría que destacar el aspecto de las cuentas anuales a presentar y el lugar de presentación, ya que si a la fecha de formulación de las cuentas anuales, aún no se ha producido la inscripción en el Registro Mercantil del proceso de transformación, la sociedad se verá obligada a presentar dichas cuentas bajo la norma contable de cooperativas y en el Registro de Cooperativas. No obstante, se podría dar el caso de que la entidad ya se encuentre dada de baja en el Registro de Cooperativas y aún no esté inscrita en el Registro Mercantil. En este caso, por sentido práctico, entendemos que la sociedad no debería formular sus cuentas bajo la norma contable cooperativa, sino bajo el PGC, independientemente de que no estuviera inscrita en el Registro Mercantil y esperar a estarlo a efectos de su presentación, ya que lo contrario no tendría sentido.

\section{ANEXOS}

\section{ANEXO 1}

\section{Informe del órgano de administración en relación con el proceso de transformación}

De acuerdo con el artículo 65 de la ley de cooperativas andaluzas, el órgano de administración preparará un informe que justifique los aspectos jurídicos y económicos de la transformación y las consecuencias que tendrá para los socios, así como el eventual impacto de género en los órganos de administración. 


\section{Aspectos jurídicos de la transformación}

El proceso de transformación que inicia la cooperativa culminará con la creación de la sociedad limitada denominada "MAPI, S.L.". Al igual que con la forma mercantil de sociedad cooperativa, los socios estarán cubiertos bajo el velo de la limitación de la responsabilidad en el capital social aportado.

En todo momento del proceso, el socio tiene su derecho de causar baja en el mismo. Las condiciones laborales se mantendrán en las mismas condiciones que se tenían en la cooperativa. Y, no existirá ningún impacto negativo en el plano de género, pues se mantendrá e incluso mejorará la participación del sexo femenino en dicho órgano.

\section{Aspectos económicos de la transformación}

Desde un punto de vista económico, los estudios de factibilidad realizados indican que el proceso de transformación permitirá una mejora en la gestión que se reflejará en un aumento del beneficio. El proceso de transformación sólo afectará la naturaleza jurídica de la entidad.

\section{ANEXO 2 \\ Informe sobre las modificaciones patrimoniales significativas que han tenido lugar una vez cerrado el balance a 15/5/2016}

Respecto al activo no corriente no se han producido diferencias significativas, produciéndose una reducción de los valores netos del inmovilizado intangible y del material, y manteniéndose el inmobiliario. La variación del activo corriente viene principalmente motivada por una reducción del efectico y de otros activos líquidos, descendiendo de 133.745 a 48.540 euros.

En lo que concierne al patrimonio neto de la sociedad, ha sufrido un considerable descenso, debido a las pérdidas acumuladas en la cooperativa durante el periodo del 1 de enero al 15 de mayo de 2016. Dichas pérdidas se corresponden a un incremento de gastos de personal en la empresa y de los servicios exteriores. Estas pérdidas, al resultar de la gestión diaria, resultan cooperativas, y, por lo tanto, son imputadas un 50\% al Fondo de Reserva Obligatorio, y el otro 50\%, al patrimonio de los socios.

El pasivo no corriente también ha disminuido ya que lo han hecho las deudas a largo plazo y los pasivos por impuesto diferido. Y, por último, el pasivo corriente se ha incrementado con motivo del aumento del saldo de los proveedores, acreedores y el personal. Aunque ha disminuido las deudas a corto plazo y las deudas con las Administraciones públicas. 


\section{Referencias Bibliográficas}

Alfonso Sánchez, R. (1997) Ámbito subjetivo de la transformación en la legislación cooperativa (algunos aspectos críticos). Revista de Derecho de Sociedades, $\mathrm{N}^{\mathrm{o}}$ 8, pp. $178-193$.

Alonso, E. (2000) La fusión, la escisión, la transformación y la extinción de las cooperativas. Boletín de la Asociación Internacional de Derecho Cooperativo, $\mathrm{N}^{\mathrm{o}} 34$, pp. $75-113$.

Bahía Almansa, B. (2007) Análisis Fiscal de la transformación de sociedades. Madrid: La Ley, grupo Wolters Kluwer.ISBN: 9788497258258.

Bahía Almansa, B. (2010) Una aproximación a la fiscalidad de la transformación en el seno de las cooperativas. GEZKI, No 6, pp. $93-119$

León Sanz, F.J. (1997) Fusión, transformación y otras modificaciones estructurales de sociedades. Revista de derecho de sociedades, No 9, pp. 25 - 60.

Macías Ruano, A. J. (2002) Consideraciones críticas sobre la fusión, escisión y transformación en la Ley 27/1999, de 16 de julio de cooperativas. REVESCO. Revista de Estudios Cooperativos, № 78, pp. 51 - 88.

Nagore, I. (2001) La transformación de la sociedad cooperativa en sociedad de responsabilidad limitada. Madrid: Dykinson. ISBN: 9788481557176.

Nagore, I. (2010) Tratamiento del Capital Social en la transformación de Cooperativas. Boletín de la Asociación Internacional de Derecho Cooperativo, No 35, pp. 47 - 50

Polo Garrido, F. (2006) La contabilidad de cooperativas en un proceso de armonización contable internacional. El caso de España. REVESCO. Revista de Estudios Cooperativos, № 89, pp. 108-138.

Rodríguez Artigas, F. (2001) Transformación de sociedades cooperativas. Revista derecho de sociedades, $\mathrm{N}^{\mathrm{o}} 17$, pp. 13 - 38.

Rodríguez Artigas, F. (2009) Modificaciones estructurales de las sociedades mercantiles. Navarra: Thomson Reuters. ISBN: 9788499034256.

Rojo Ramírez, A.; Esteban Cerdán, A.; Sánchez Pérez, J.A. (2001) La transformación de cooperativas. Especial referencia al caso andaluz. Ciriec-España, Revista de Economía Pública, Social y Cooperativa, $\mathrm{N}^{\mathrm{o}} 38$, pp. 57-76.

Vidal Portabales, J. I. (1997) La transformación de la sociedad cooperativa en el proyecto de Ley Gallega de cooperativas. Dereito, No 2, Vol. 6, pp. 217 - 248.

\section{Legislaciones}

Ley 3/1987, de 2 de abril, General de Cooperativas (BOE $n^{\circ} 84$, de 8 de abril).

Ley 13/1989, de 26 de mayo, de Cooperativas de Crédito (BOE $\mathrm{n}^{\circ} 129$, de 31 de mayo).

Ley 19/1989, de 25 de julio, de reforma parcial y adaptación de la legislación mercantil a las directivas de la comunidad Económica Europea (CEE) en materia de sociedades. (BOE ${ }^{\circ} 178$, de 27 de julio).

Ley 20/1990, de 19 de diciembre, sobre Régimen Fiscal de las Cooperativas (BOE ${ }^{\circ}$ 304, de 20 de diciembre).

Ley 2/1995, de 23 de marzo, de Sociedades de Responsabilidad Limitada (BOE nº71, de 24 de marzo).

Ley 2/1999, de 31 de marzo, de Sociedades Cooperativas Andaluzas (BOE n 107, de 5 de mayo).

Ley 27/1999, de 16 de julio, de cooperativas (BOE $\mathrm{n}^{\circ} 170$, de 17 de julio). 
Ley $3 / 2009$, de 3 de abril, sobre modificaciones estructurales de las sociedades mercantiles $\left(\mathrm{BOE}^{\circ} 82\right.$, de 4 de abril)

Ley 14/2011, de 23 de diciembre, de Sociedad Cooperativas Andaluzas (BOE n 17, de 20 de enero).

Ley 5/2011, de 29 de marzo, de Economía Social (BOE nº 76, de 11 de marzo)

Ley 27/2014, de 27 de noviembre, del Impuesto sobre Sociedades. (BOE n⿳⺈ 288, de 28 de noviembre de 2014)

Real Decreto 888/1969, de 9 de mayo, por el que se promulga el nuevo Estatuto fiscal de las Cooperativas (BOE $\mathrm{n}^{\circ} 114$, de 13 de mayo).

Real Decreto 2396/1971, de 13 de agosto, por el que se aprueba el Reglamento de Cooperación (BOE No242, de 9 de octubre de 1971).

Real Decreto 530/1973, de 22 de febrero, por el que se aprueba el plan general de contabilidad (BOE $\mathrm{n}^{\circ} 79$, de 2 de abril).

Real Decreto 1643/1990, de 20 de diciembre, por el que se aprueba el Plan General de Contabilidad. (BOE $n^{\circ} 310$, de 27 de diciembre).

Real Decreto 1784/1996, de 19 de julio, por el que se aprueba el Reglamento del Registro Mercantil.

Real Decreto 1514/2007, de 16 de noviembre, por el que se aprueba el Plan General de Contabilidad (BOE $\mathrm{n}^{\circ} 278$, de 20 de noviembre).

Real Decreto 1515/2007, de 16 de noviembre, por el que se aprueba el Plan General de Contabilidad de Pequeñas y Medianas Empresas y los criterios contables específicos para microempresas (BOE n²79, de 21 de noviembre).

Real Decreto 267/2011, de 11 de diciembre, por el que se aprueba el Reglamento de desarrollo de la Ley de Sociedades Cooperativas Andaluzas en materia registral y de autorizaciones $\left(\mathrm{BOE} \mathrm{n}^{\circ} 24\right.$, de 26 de febrero).

Real Decreto 123/2014, de 2 de septiembre, por el que se aprueba el Reglamento de la Ley 14/2011 (BOJA n ${ }^{\circ} 186$, de 23 de septiembre).

Decreto Legislativo 1/2010, de 2 de julio, por el que se aprueba el texto refundido de la Ley de Sociedades de Capital (BOE no 161, de 3 de julio).

Orden EHA/3360/2010, de 21 de diciembre, por la que se aprueban las normas sobre los aspectos contables de las Sociedades Cooperativas (BOE n³16, de 29 de diciembre). 